\title{
Data integration for identification of important transcription factors of STAT6-mediated cell fate decisions
}

\author{
M. Jargosch ${ }^{1 *}$, S. Kröger ${ }^{2 *}$, E. Gralinska ${ }^{1}$, U. Klotz ${ }^{1,3}$, Z. Fang ${ }^{1}$, W. Chen ${ }^{4}$, \\ U. Leser ${ }^{2}$, J. Selbig ${ }^{3}$, D. Groth ${ }^{3}$ and R. Baumgrass ${ }^{1}$ \\ ${ }^{1}$ Signal Transduction, German Rheumatism Research Center (DRFZ), Berlin, \\ Germany \\ ${ }^{2}$ Knowledge Management in Bioinformatics, Humboldt-Universität zu Berlin, \\ Berlin, Germany \\ ${ }^{3}$ Bioinformatics Group, University of Potsdam, Potsdam, Germany \\ ${ }^{4}$ Scientific Genomics Platform, Berlin Institute for Medical Systems Biology, \\ Max Delbrück Center for Molecular Medicine, Berlin, Germany \\ *These authors contributed equally to this study. \\ Corresponding author: R. Baumgrass \\ E-mail: baumgrass@drfz.de \\ Genet. Mol. Res. 15 (2): gmr.15028493 \\ Received January 26, 2016 \\ Accepted March 17, 2016 \\ Published June 24, 2016 \\ DOI http://dx.doi.org/10.4238/gmr.15028493
}

\begin{abstract}
Data integration has become a useful strategy for uncovering new insights into complex biological networks. We studied whether this approach can help to delineate the signal transducer and activator of transcription 6 (STAT6)-mediated transcriptional network driving $\mathrm{T}$ helper (Th) 2 cell fate decisions. To this end, we performed an integrative analysis of publicly available RNA-seq data of Stat6knockout mouse studies together with STAT6 ChIP-seq data and our own gene expression time series data during Th2 cell differentiation. We focused on transcription factors (TFs), cytokines, and cytokine receptors and delineated 59 positively and 41 negatively STAT6-regulated genes,
\end{abstract}


which were used to construct a transcriptional network around STAT6. The network illustrates that important and well-known TFs for Th2 cell differentiation are positively regulated by STAT6 and act either as activators for Th2 cells (e.g., Gata3, Atf3, Satb1, Nfil3, Maf, and Pparg) or as suppressors for other Th cell subpopulations such as Th1 (e.g., Ar), Th17 (e.g., Etv6), or iTreg (e.g., Stat3 and Hifla) cells. Moreover, our approach reveals 11 TFs (e.g., Atf5, Creb3l2, and Asb2) with unknown functions in Th cell differentiation. This fact together with the observed enrichment of asthma risk genes among those regulated by STAT6 underlines the potential value of the data integration strategy used here. Thus, our results clearly support the opinion that data integration is a useful tool to delineate complex physiological processes.

Key words: Data integration; Th2 cells; Gene regulatory network; STAT6; Transcription factors

\section{INTRODUCTION}

The development of microarray technologies and next-generation sequencing (NGS) methods in the last decades has transformed modern biology (Schuster, 2008). Thousands of high-throughput studies with manifold research foci have resulted in an explosion of heterogeneous omics data. Public online databases such as GEO for microarray and NGS data (Edgar et al., 2002), TRANSFAC for transcription factors (TFs) and their DNA binding sites (Wingender et al., 1996), or tranSMART for clinical and translational research (Athey et al., 2013) ensure long-term access. The availability of these omics data encourages their reuse-even in another context. However, these data are derived from different technological platforms, have distinct formats, and come from diverse sources, which challenge their uniform processing and integrated analysis. To this end, comprehensive data integration approaches are urgently needed.

Data integration strives for the usage of multiple omics datasets from distinct methods such as gene expression analysis through microarray or RNA-seq, ChIP-seq, mass spectrometry, genome-wide single-nucleotide polymorphism (SNP) genotyping, and GWAS. Moreover, data integration allows use of data of the same type but from various sources with multiple formats. Several reviews and general comments have predicted that the integration of omics data and clinical data would facilitate identification of novel biomarkers of diseases and candidate targets for therapeutic approaches. All these could help to increase the efficiency of drug discovery (Searls, 2005; Slater et al., 2008; Bielekova et al., 2014; Subramanian et al., 2015).

Consequently, data integration has been applied extensively in biomedical research over the previous decades, e.g., to study the functional and structural relationships of genes for comprehensive network analysis. Recently, Subramanian et al. (2015) summarized the potential of data integration and network analysis for uncovering physiological processes of immune cells. For instance, in macrophages, gene expression dynamics and scanning for TF-binding sequence motifs have been used to elucidate transcriptional networks on a large scale (Ramsey et al., 2008; Litvak et al., 2012), while a combination of genome-wide mRNA expression data and network perturbation using methods such as RNAi knockdown was applied to identify useful intervention strategies in infections (König et al., 2010). In T helper (Th) 
cells, Ciofani et al. (2012) demonstrated the power of data integration to construct a regulatory network for Th17 cell differentiation: they applied an integrative approach to delineate the Th17 cell global transcriptional regulatory network using meta-analysis of genome occupancy of multiple TFs, RNA-seq data of TF-deficient T cells, and immune cell transcriptome data. With this approach, they identified the TFs BATF and IRF4 as key activators and FOSL2 as a modulator of Th17 cell differentiation.

In this work, we used data integration as a tool for understanding the Th cell fate decisions of naïve Th cells during differentiation, specifically focusing on Th2 cells. The differentiation of naïve $\mathrm{CD}^{+} \mathrm{T}$ cells into Th cell subtypes, such as Th2, Th1, Th17, and induced $\mathrm{T}$ regulatory (iTreg) cells, is regulated by specific cytokine milieus and complex TF networks. The cytokine interleukin 4 (IL4) activates signal transducer and activator of transcription 6 (STAT6) and initiates the differentiation of Th2 cells. Th2 cells themselves secrete the signature cytokines IL4, IL5, and IL13 and express the master TF GATA3 (Yamane and Paul, 2013). However, cooperation among TFs to determine the specific program for Th2 cell differentiation is poorly understood. Nevertheless, it is of high medical importance because Th2 cells play a critical role in the pathogenesis of allergies and asthma (Del Prete, 1992; Kay, 2001).

To identify critical TFs and cytokines for Th2 cell differentiation, we used an integrative strategy combining global gene expression and DNA-binding data for Th2 cells from wild-type (WT) and Stat6-knockout (KO) mice and constructed a global gene regulatory network for Th2 cells around the TF STAT6. We discovered that TFs, which are important for Th2 cell differentiation, are positively regulated by STAT6 and act either as activators for Th2 cells or as suppressors for Th1, Th17, or iTreg cells. Additionally, we observed a Th2-cell subtype-specific expression preference of the STAT6-positively regulated TFs and an enrichment of asthma risk genes within STAT6-regulated genes. Finally, we identified 11 STAT6-regulated and -bound TFs with unknown functions in Th cell differentiation. Further experiments are needed to examine whether these TFs are indeed crucial for Th2 cell differentiation and to assess their potential for therapeutic manipulations. The comprehensive strategy used here illustrates the power of integrating global gene and DNA binding data for construction of transcriptional networks in Th2 cell differentiation and supports the opinion that data integration is a useful tool to identify novel biological mechanisms.

\section{RESULTS AND DISCUSSION}

\section{Construction of a network of STAT6-regulated genes in Th2 cells}

To comprehensively identify TFs acting as regulatory hubs in Th2 cell differentiation, we performed an integrated analysis of four publicly available global gene expression and DNA-binding datasets for Th2 cells and one in-house generated gene expression dataset (Figure 1A). The datasets are displayed in Figure 1: RNA-seq data for Stat6-KO mice, microarray data for Th cells, STAT6 ChIP-seq data, and RNA-seq gene expression time series for Th cells. The applied integration strategy enabled derivation of a gene regulatory network around the TF STAT6, which fundamentally influences the cell fate decision towards the Th2 cell subtype (Yamane and Paul, 2013).

First, we identified STAT6-regulated genes by analyzing RNA-seq data for 7-day-old in vitro differentiated Th2 cells of WT and Stat6-KO mice (Figure 1, green) (Vahedi et al., 
2012), which yielded 1047 differentially expressed genes. These presumed STAT6-regulated genes were filtered for TFs, cytokines, and cytokine receptors based on a published Th cell microarray dataset (Figure 1, purple) of 782 TFs and 271 cytokines and cytokine receptors in the context of induction, differentiation, and maintenance of Th cell subsets (Wei et al., 2009). The resulting 100 genes (Figure 1B) formed the basis of the STAT6 network derived in the subsequent steps. These 100 genes were compared to STAT6 ChIP-seq data for 7-day-old Th2 cells (Figure 1, orange) (Wei et al., 2010) to discriminate between direct and indirect targets of STAT6 (Figure 1B). Finally, we integrated expression time series of Th2 cells (Hu et al., 2013) (in-house generated RNA-seq expression data of Th2 cells, manuscript in preparation: Jargosch M., Hoang Y., Kröger S., Fang Z., Chen W., Baumgrass R.) to characterize these candidates with respect to their expression behavior compared to naïve $\mathrm{CD} 4^{+} \mathrm{T}$ cells in the early $(0-48 \mathrm{~h})$ and late $(72-336 \mathrm{~h})$ stages of Th2 cell differentiation.

A
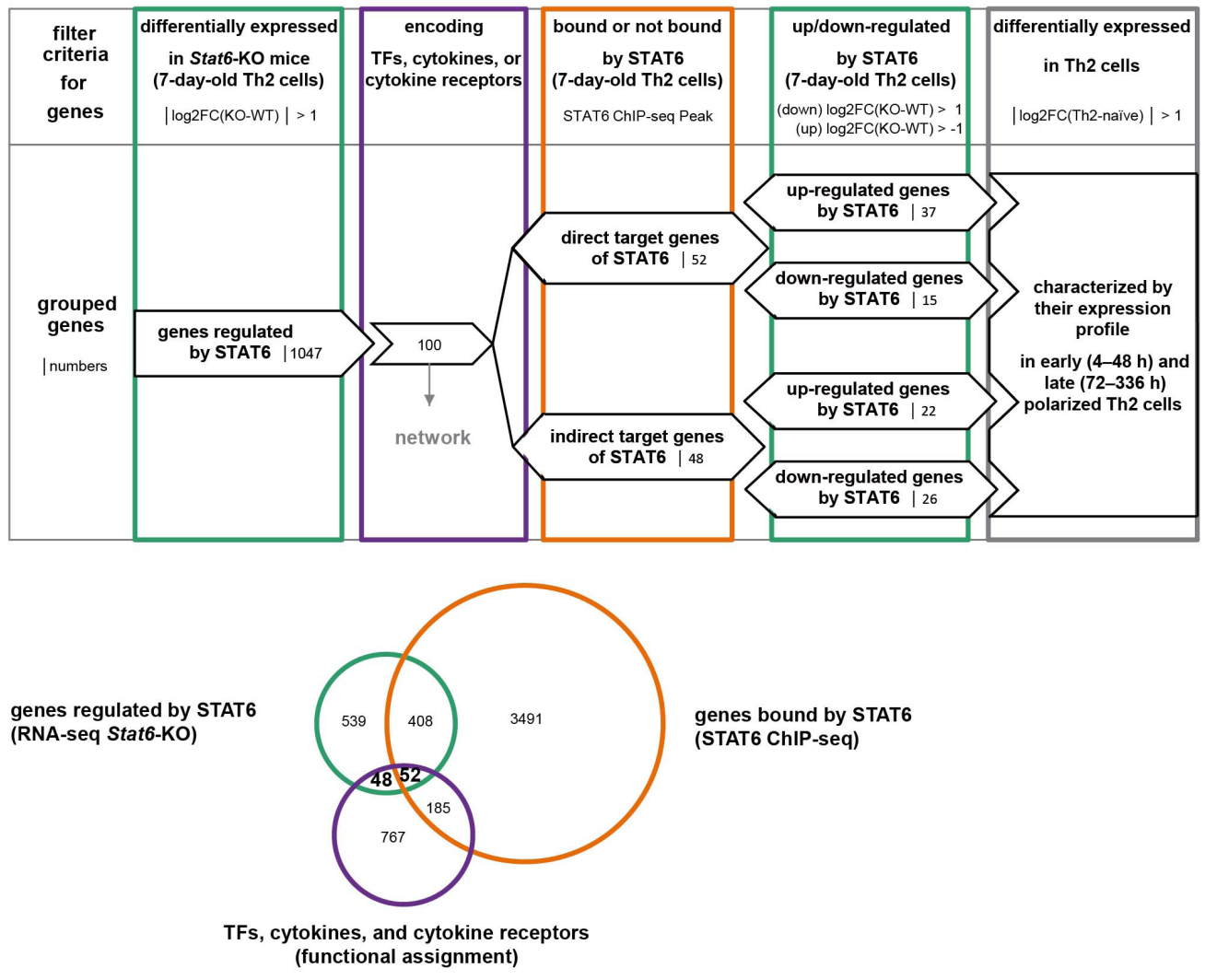

Figure 1. Strategy of data integration. A. Workflow for the construction of the STAT6 network. The filter criteria and datasets used for each step are shown in the first row and highlighted in different colors. The filter criteria and/ or the number of genes are listed for each integration step and gene group in the second row. B. The Venn diagram shows the three datasets forming the basic network. The intersection (100 genes, highlighted bold) of STAT6regulated genes (green) with TFs, cytokines, and cytokine receptors (purple) are dissected into direct and indirect target genes of STAT6 by integrating the STAT6 ChIP-seq data (orange). 
The resulting STAT6 network shown in Figure 2 contains the following information: i) how STAT6 influences the expression of other genes, ii) whether genes encode TFs or cytokines/cytokine receptors, iii) whether gene regions are directly bound by STAT6, and iv) whether genes are differentially expressed in early and/or late differentiation of Th2 cells compared to naïve $\mathrm{CD}^{+} \mathrm{T}$ cells. The network in Figure 2 depicts the genes in a four-ring structure around STAT6 based on their expression behavior during the early and late stages of Th2 cell differentiation.

The gene regulatory network includes $100 \mathrm{TFs}$, cytokines, and cytokine receptors in Th2 cells that are regulated by (48 genes) or even bound by STAT6 (52 genes) (Figure 2). Altogether, 59 and 41 genes are up- and down-regulated by STAT6, respectively. The integration of expression time series of Th2 cells revealed that 98 of these 100 genes are also differentially expressed in polarized $\mathrm{Th} 2$ cells compared to naïve $\mathrm{CD} 4^{+} \mathrm{T}$ cells, with most differentially expressed in both the early and late phases of differentiation. The expression of 52 and 26 genes is increased and decreased in Th2 cells, respectively, where 20 genes show opposite expression in the early and late phases.

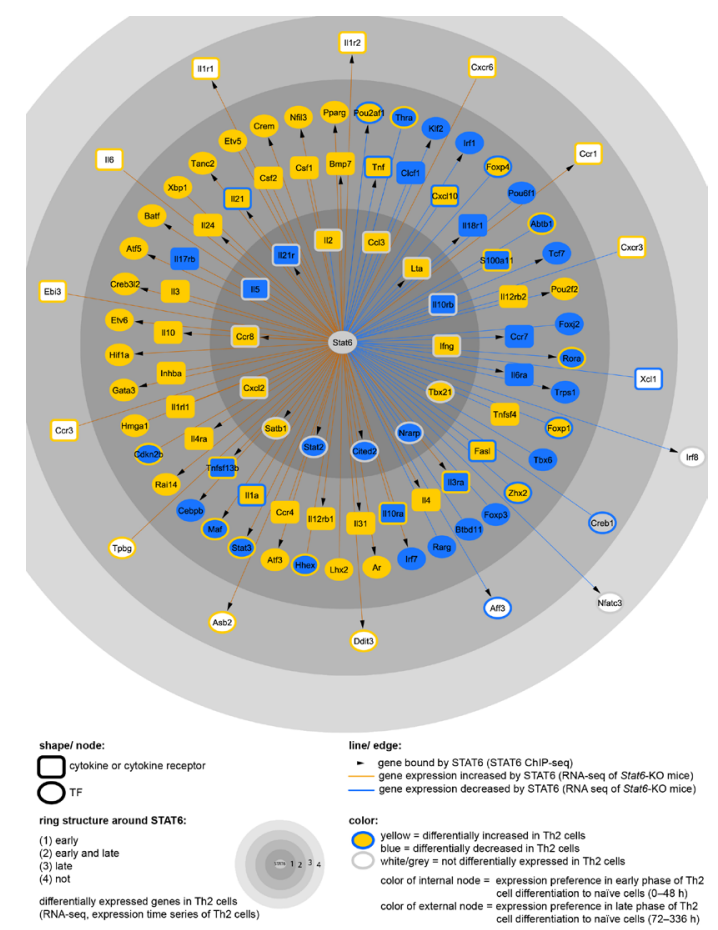

Figure 2. Gene regulatory network of STAT6-regulated genes in Th2 cells. The network is focused on TFs (squares) and cytokines or cytokine receptors (ovals) and shows indirect (48 genes) and direct targets (52 genes) of STAT6. The edge color indicates the effect of STAT6 on gene expression (blue $=$ down-regulated, yellow $=$ upregulated). Arrowheads indicate direct binding by STAT6. The node color (blue = differentially decreased, yellow $=$ differentially increased, white/grey $=$ not differentially expressed) characterizes the expression of these genes in Th2 cells compared to naïve $\mathrm{CD}^{+} \mathrm{T}$ cells (inside color $=$ early phase, outline color $=$ late phase). The network layout is based on four rings around STAT6 in graded grey tones. Rings from inside to outside contain genes according to their differential expression behavior in the early (1), early and late (2), or late (3) phases or lack of differential expression (4) in Th2 cells compared to naïve cells. 
The network contains important genes known to be active in Th2 cells, such as the master TF of Th2 cells, Gata3; the Th2 cell-specific surface receptors Ccr3, Ccr4, and Ccr8; and the cytokines Il4 and Il6, which are positively regulated by STAT6 (Bonecchi et al., 1998; D’Ambrosio et al., 1998; Yamane and Paul, 2013). Importantly, master TFs for other Th cell subsets are included but negatively regulated by STAT6, such as Foxp3 in iTreg cells, Tbx21 in Th1 cells, and Rorg in Th17 cells (Yamane and Paul, 2013).

\section{Th2 cell-specific TFs are positively regulated by STAT6 and increased in Th2 cells}

It is well accepted that networks of multiple TFs are required to execute a full differentiation program and that many of them act as activators or repressors in a contextdependent manner (Novershtern et al., 2011). Next, we studied the function of STAT6regulated and STAT6-bound genes encoding TFs in Th cell differentiation processes and identified Th2 cell-specific TFs as activators for Th2 differentiation or repressors for Th1, Th17, or iTreg cell differentiation. We filtered the 100 STAT6-regulated genes for TFs and performed a literature search regarding their function in differentiation processes. Their main functions in Th cell differentiation and their regulation by STAT6 in Th2 cells are summarized in Table 1. The 32 TFs were divided into three groups: i) associated with differentiation of Th2 cells, ii) associated with differentiation of other Th cell subtypes, and iii) previously unknown association with differentiation of Th cells. Most Th2 cell-specific TFs were up-regulated by STAT6. Consistently, the majority of STAT6-negatively regulated TFs were activators for Th1, Th17, or iTreg cell differentiation. Interestingly, there were only two TFs acting as repressors of Th2 cell differentiation within the STAT6-negatively regulated TFs (Nfatc3 and Irf1). Thus, STAT6-driven differentiation of Th2 cells is mainly modulated by activators and not preferentially by repressors.

The 32 STAT6-regulated TFs were included into a literature search concerning the context of T cell differentiation within the publication period 2000-2015. Depending on the discovered function in differentiation processes the TFs were divided into three groups: i) associated with differentiation of Th2 cells, ii) associated with differentiation of other Th cell subtypes and iii) not associated with differentiation of Th cells. The list was supplemented with the information how the gene expression of each TF is regulated by STAT6 and how their differential expression pattern is directed in early and late polarized Th2 cells compared to naïve T cells ( $\uparrow=$ increased (yellow), $\downarrow=$ decreased (blue) and n.diff $=$ not differentially expressed).

Six TFs were associated with the differentiation of Th2 cells: Gata3, Atf3, Satb1, Nfil3, Maf, and Pparg; these TFs were positively regulated by STAT6 and increased in Th2 cells. The expression of Gata3, Satb1, Nfil3, Maf, and Pparg was previously shown to be increased by STAT6 in primary human CD4+ T cells by blocking STAT6 with RNAi (Elo et al., 2010). Additionally, IL4 is known to increase the expression of Atf3 and Satb1 (Chen et al., 2003b; Ahlfors et al., 2010). Furthermore, SATB1 coordinates Th2 cell differentiation by positively regulating Gata3 (Notani et al., 2010). SATB1, NFIL3, MAF, and PPARG positively regulate the gene expression of the Th2 cytokine Il4 (Ribeiro de Almeida et al., 2009; Kashiwada et al., 2011; Lai et al., 2012; da Rocha Junior et al., 2013; Kwon et al., 2014). In addition to the positive effects on Th2 cell differentiation, NFIL3 suppresses Th17 cell development by directly binding and repressing the Rorg promoter (Yu et al., 2013). PPARG is also known to suppress Th17 and Th1 cell differentiation by decreasing the level of IFN $\gamma$ production in splenocytes (da Rocha Junior et al., 2013). 
STAT6 network of Th2 cells

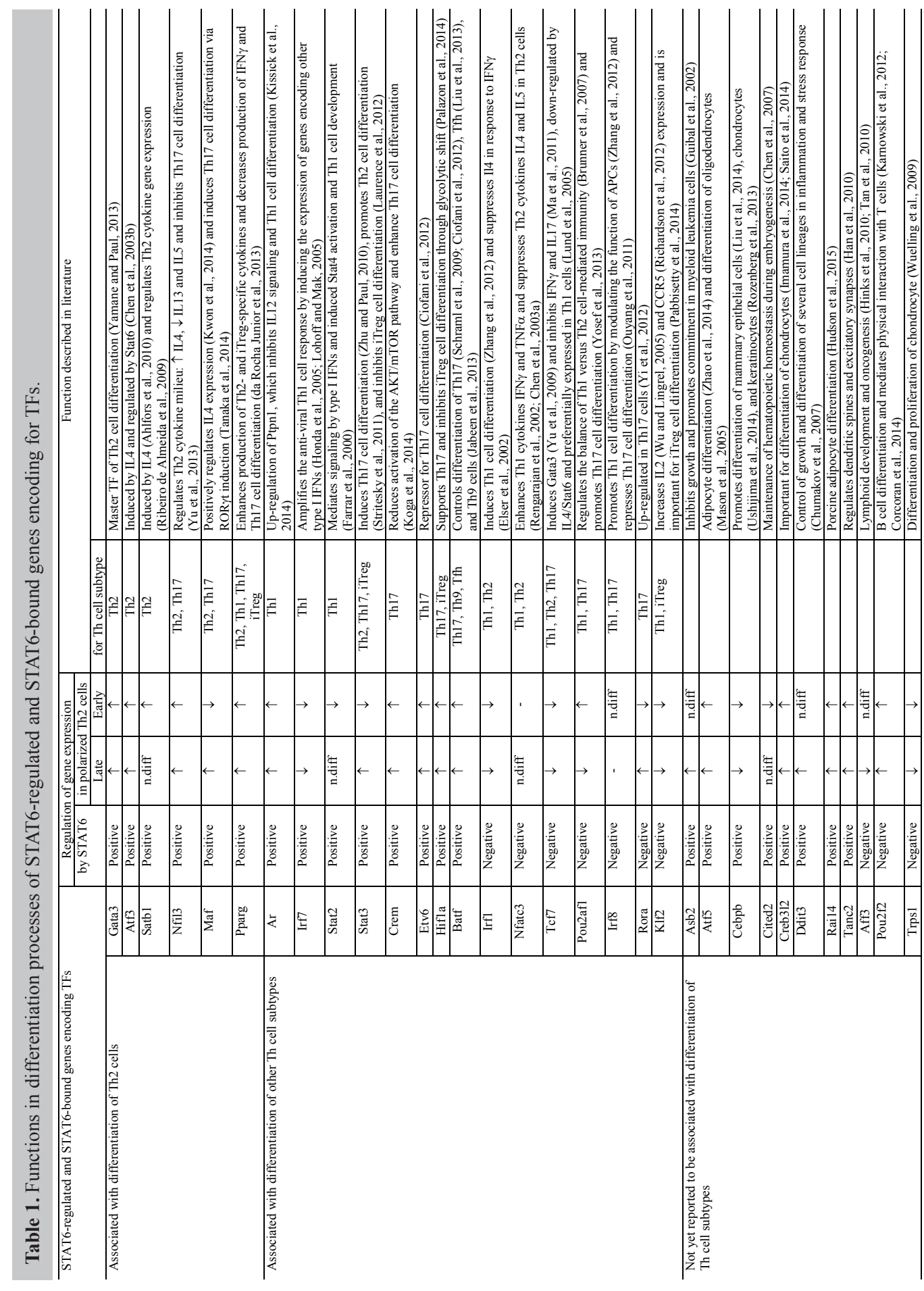


Fifteen TFs were associated with the differentiation of other Th cell subtypes without a reported main function in Th2 cell differentiation. Of these, eight (Ah, Stat3, Etv6, Hifl $\alpha$, Irf7, Stat2, Batf, and Crem) were increased, and seven (Irf1, Irf8, Nfatc3, Pou2af1, Rora, $K l f 2$, and $T c f 7$ ) were decreased by STAT6. In summary, STAT6-negatively regulated TFs were positive regulators for other Th cell populations using different modes of action. The TFs IRF8, IRF1, and NFATC3 enhance Th1 cell differentiation by modulating the function of APCs (Taki et al., 1997; Salkowski et al., 1999; Maruyama et al., 2003; Lohoff and Mak, 2005; Zhang et al., 2012) or directly promoting the expression of Th1 cytokines Ifng and $\operatorname{Tnf}$ (Chen et al., 2003a). The TFs POU2AF1 and ROR $\alpha$ promote Th17 cell differentiation (Yi et al., 2012; Yosef et al., 2013), and KLF2 is necessary for the generation of iTreg cells (Pabbisetty et al., 2014). Additionally, IRF1 and NFATC3 are known as suppressors for Th2 cytokines (Elser et al., 2002; Rengarajan et al., 2002; Chen et al., 2003a). Consistent with this, a few TFs were increased by STAT6 and associated with suppressive processes in Th1, Th17, or iTreg cells. For instance, AR inhibits IL12 signaling and Th1 cell differentiation by up-regulating Ptpn1 (Kissick et al., 2014), STAT3 activation and HIF1 $\alpha$ inhibit iTreg cell differentiation (Laurence et al., 2012; Palazon et al., 2014), and ETV6 is known as a repressor in Th17 cells (Ciofani et al., 2012). However, STAT3 can also induce Th17 cell differentiation (Zhu and Paul, 2010) and promote Th2 cell differentiation together with IL6 (Stritesky et al., 2011).

Another 11 TFs were previously unreported in the context of Th cell differentiation. However, often they are associated with the differentiation processes of other cell subtypes such as B cells, dendritic cells, chondrocytes, and adipocytes (vide infra).

In summary, our analyses of the literature confirm the value and highlight the explanatory power of the STAT6 network. Furthermore, they confirm that important TFs for Th2 cell differentiation are up-regulated in the STAT6 network and acting either as activators for Th2 cells or suppressors for Th1, Th17, or iTreg cells.

\section{Most of the STAT6-positively regulated genes are preferentially expressed in Th2 cells}

To validate the Th2 cell specificity of the STAT6 network, we next studied gene expression preferences in Th cells for the 100 STAT6-regulated genes. To evaluate the hypothesis that STAT6-positively regulated genes have higher expression in Th2 cells than STAT6-negatively regulated genes, we compared the gene expression levels for each of the 100 STAT6-regulated genes in 10-day-old in vitro differentiated Th1, Th2, Th17, and iTreg cells (Wei et al., 2009). We identified 64 genes preferentially expressed in one Th-cellsubtype. We subdivided these genes into Th1-, Th2-, Th17-, and iTreg-specific genes and discriminated between STAT6-positively (35) and STAT6-negatively regulated (29) genes (Figure 3A). Most of the 35 STAT6-positively regulated genes (22) were preferentially expressed in Th2 cells. Importantly, there were no Th2 subtype-specific genes among the STAT6-negatively regulated genes, validating the Th2 cell specificity of the STAT6 network. Furthermore, these results support the observation that Th2-subtype-specific genes were upregulated by STAT6, whereas Th1-, Th17-, or iTreg-subtype-specific genes were mainly down-regulated by STAT6. 

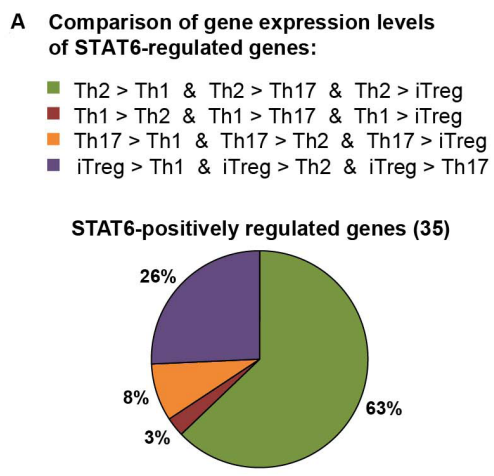

STAT6-negatively regulated genes (29)

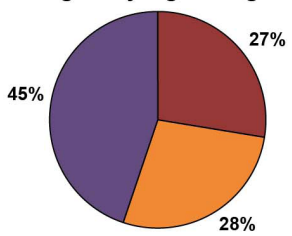

B SNF

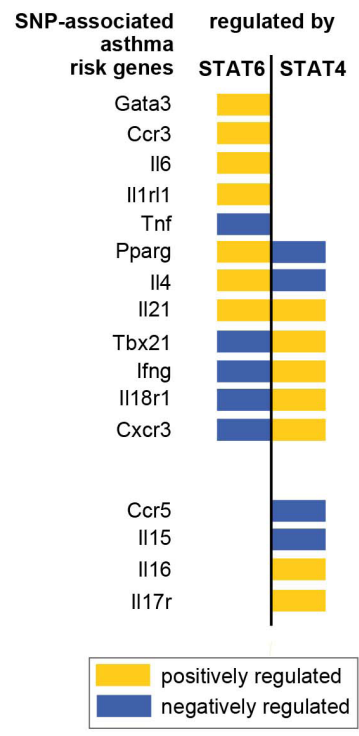

Number of asthma risk genes within STAT6- and STAT4-regulated genes:

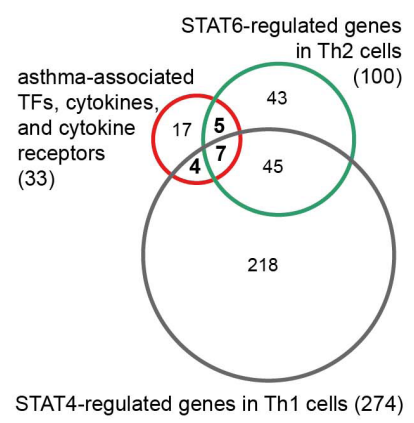

STAT4-regulated genes in Th1 cells (274)

Figure 3. STAT6-positively regulated genes are specific for the Th2 cell phenotype and are associated with asthma risk genes. A. Expression preferences of Th cell subtype-specific and STAT6-regulated genes are shown. Gene expression levels of 100 STAT6-regulated genes were compared between the Th cell subtypes Th1, Th2, Th17, and iTreg. B. Right: The Venn diagram highlights the intersection of SNP-associated asthma risk genes encoding TFs, cytokines, or cytokine receptors with STAT6-regulated genes in Th2 cells and with STAT4-regulated genes in Th1 cells. Left: Asthma risk genes within STAT6-regulated genes in Th2 cells and/or STAT4-regulated genes in Th1 cells are depicted. STAT6 or STAT4 effects on gene expression regulation are shown (yellow bars = up-regulated, blue bars $=$ down-regulated) .

\section{STAT6-regulated genes are associated with asthma risk genes}

Hyper-activated $\mathrm{CD}^{+} \mathrm{T}$ cells play an important role in allergic asthma and various autoimmune diseases such as rheumatoid arthritis. Allergic asthma is associated with an infiltration of Th2 cells, whereas autoimmune diseases are driven by Th1 and Th17 cells (Dolhain et al., 1996; Kay, 2001). Therefore, we suspected an enrichment of asthma risk genes within the STAT6-regulated genes in Th2 cells compared to STAT4-regulated genes in Th1 cells. Indeed, the comparison of asthma risk genes (Renkonen et al., 2010) with the datasets of STAT6-regulated genes in Th2 cells and STAT4-regulated genes in Th1 cells showed an enrichment of asthma risk genes in the set of STAT6-regulated genes.

Recently, asthma risk genes were identified by genome-wide SNP association studies. A meta-analysis of SNPs by Renkonen et al. (2010) identified 125 asthma-associated genes. Filtering these risk genes for TFs, cytokines, and cytokine receptors (microarray data for Th cells, Wei et al., 2009) yielded 33 asthma risk genes (Figure 3B, red), which were integrated with the 100 STAT6-regulated genes in Th2 cells (Figure 3B, green) and the 274 STAT4regulated genes in Th1 cells (Figure 3B, gray). Similar to the STAT6-regulated genes, the STAT4-regulated genes were identified by analyzing RNA-seq data for 7-day-old in vitro differentiated Th1 cells of WT and Stat4-KO mice (Vahedi et al., 2012). 
The analysis revealed that 12 of the 100 STAT6-regulated genes were asthma risk genes. Of these genes seven and five were positively and negatively regulated by STAT6, respectively. Among the positively regulated asthma risk genes (Figure 3B, yellow bars), there were Th2-subtype-specific genes such as Gata3, Ccr3, Il6, Il4, Illrll, and Pparg, which are also preferentially expressed in Th2 cells (microarray data of Th cells, Wei et al., 2009). Consistently, the negatively regulated asthma risk genes (Figure 3B, blue bars) were the Th1subtype-specific genes $T b x 21$, Ifng, Tnf, Il18r1, and Cxcr3. Unexpectedly, we also revealed 11 asthma risk genes within the STAT4-regulated genes in Th1 cells; however, almost all of these were regulated oppositely by STAT4 in Th1 cells compared to STAT6 in Th2 cells.

Our results support the common view that asthma is associated with an imbalanced Th1/Th2 cell ratio. Recently, it was demonstrated in an airway inflammation model that the frequency of Th2 cells and the absolute amounts of Th2 cytokines and actived STAT6 TF were increased. Consequently, Th1 cytokines and the activated STAT4 TF are decreased in this model (Chen et al., 2015).

In summary, this analysis validates the Th2 cell specificity of the STAT6 network and confirms that the STAT6 network includes genes that are positively associated with the Th2 cell differentiation and up-regulated by STAT6 as well as genes that are positively associated with the differentiation of Th1, Th17, or iTreg cells and that are mostly down-regulated by STAT6.

\section{Novel and potentially important TFs for Th2 cell fate decisions}

We applied the described data integration strategy to better understand Th2 cell fate decisions and, in particular, to delineate the gene regulatory network of $\mathrm{Th} 2$ cells to unravel novel important TFs in Th2 cell differentiation. We identified 11 direct target genes of STAT6 with previously unreported functions in Th cell differentiation. Among these were eight (Asb2, Atf5, Creb3l2, Cebpb, Cited2, Rai14, Tanc2, and Ddit3) and three genes (Aff3, Trps1, and Pou 2 2) positively and negative regulated by STAT6, respectively (Table 1).

The TFs AFF3 and POU2F2 have not been directly associated with Th cell differentiation processes; however, they have been connected with Th1 cells. Aff3 is a rheumatoid arthritis susceptibility gene that also influences the response to anti-TNF treatment (Tan et al., 2010). The function of POU2F2 in B cell differentiation was extensively studied and shown to mediate the physical interaction of B and T cells (Corcoran et al., 2014); however, its role in Th cell differentiation has not been described. In our network, the expression of Pou $f 2$ was increased in the early and late stages of Th2 cell differentiation compared to naïve T cells. However, Cron et al. (2001) reported that POU2F2 decreases $I l 4$ promoter activity and increases $I l 2$ promoter activity. Therefore, POU2F2 promotes a Th1 pattern rather than a Th2 pattern of cytokine gene expression by activated $\mathrm{CD}^{+} \mathrm{T}$ cells. This observation was confirmed by gene expression data from Hu et al. (2013) reporting a twofold higher expression of Pou2f2 in Th1 cells than in Th2 cells.

The eight STAT6-positively regulated and previously unidentified Th2 subtyperelated TFs (Table 1) are of particular interest for us because these TFs act as activators in Th2 differentiation or suppressors in Th1, Th17, or iTreg differentiation, as shown for the other identified genes (Table 1). Interestingly, the TFs ATF5 and CREB3L2 are described as transcriptional activators of differentiation processes of adipocytes (Zhao et al., 2014) and chondrocytes (Imamura et al., 2014; Saito et al., 2014), activating transcription by binding to the cAMP response element (Umemura et al., 2015). The TF ASB2 was identified as a growth inhibitor in myeloid leukemia cells (Guibal et al., 2002) through promoting ubiquitination 
of Notch targets such as E2A and JAK2 (Nie et al., 2011). In human T cells, E2A has been described as a positive regulator of Gata3 by displacement of the repressor ZEB (Grégoire and Roméo, 1999). Interestingly, we observed that Atf5, Creb3l2, and Asb2 were differentially up-regulated in both the early and late phase of Th2 cell differentiation. Currently, we are examining whether these TFs act as regulatory hubs in Th2 cell differentiation using perturbation methods and whether they are useful for manipulation of asthma and/or allergic diseases in animal models.

Our results show that an integration of publicly available and newly collected datasets with different sources, formats, applied analysis methods, and evaluation priorities is useful for discovering new contexts within biological networks for experimental validation.

\section{MATERIAL AND METHODS}

\section{Preparation and analysis of publicly available data for gene regulatory network reconstruction}

We used selected public datasets to reconstruct gene regulatory networks, namely RNA-seq (Vahedi et al., 2012; Hu et al., 2013), microarray (Wei et al., 2009), and ChIPseq (Wei et al., 2010) datasets. In addition, we generated an RNA-seq data for Th2 cell differentiation. GEO accession numbers and corresponding references are listed in $\underline{\mathbf{S 1} \text { Table. }}$

The initial network was established based on a Stat6-KO mouse RNA-seq experiment by Vahedi et al. (2012) (GSE40463) using published normalized fpkm values to identify differentially expressed genes. Differential expression was defined as an absolute $\log 2$ (fold change) $>1$. Low-expressed genes were removed (normalized fpkm $<1$ in all groups).

We retrieved and integrated raw STAT6 ChIP-seq data for WT and STAT6-deficient Th2 cells (GSM550311, GSM550312 part of GSE22105) (Wei et al., 2010). Reads were mapped to mouse genome (mm9) using BWA (Burrows-Wheeler Aligner) (Li and Durbin, 2009). To detect TF binding sites, we applied CisGenome (Ji et al., 2008) peak detection software to all uniquely mapped reads. As described by Wei et al. (2010), ChIP-seq peaks present in KO and WT cells were removed before TF binding analysis.

The RNA-seq experiment conducted by Hu et al. (2013) (GSE22081) was analyzed based on the available raw data to ensure compatibility with our in-house performed RNA-seq experiments (described below). Ensembl genome assembly mm10 was used for annotation of sequenced reads; for both datasets, the same tools were applied for mapping, annotation, and expression analysis. After retrieving raw data from the GEO repository, reads were mapped and indexed using tophat2 (Kim et al., 2013) and SAMtools (Li et al., 2009). Detected reads per gene (gene counts) were calculated using HTSeq (Anders et al., 2015), and then differential expression was detected with R package DESeq (Anders and Huber, 2010). Thresholds for differentially expressed genes were defined by an absolute $\log 2$ (fold change) $\geq 1$ and $\mathrm{P}<0.05$. The resulting set of genes was used for further analysis and network construction. $\underline{\text { S2 Table }}$ lists all gene expression measurements and corresponding $\log 2$ (fold change) values used to construct the global gene regulatory STAT6 network.

\section{Processing RNA-seq experiments for Th2 cells}

For the differentiation cultures, BALB/cAnNCrl mice were obtained from Charles 
River Laboratories, Wilmington, Massachusetts, USA). Mice were bred under pathogen-free conditions. The animal experiment was performed according to state guidelines and approved by the local Ethics Committee LAGeSo (Landesamt für Gesundheit und Soziales) Berlin within the frame of animal experiment license T0187-01.

Naïve $\mathrm{CD} 4^{+} \mathrm{CD} 25^{-} \mathrm{CD} 45 \mathrm{RB}^{\text {high }} \mathrm{T}$ cells from spleen and lymph node cell suspensions were isolated by FACS using an Aria II sorter after surface staining with antibodies targeting CD4 (clone GK1.5), CD25 (clone PC61.5), and CD45RB (clone C363.16A). The culture medium used was RPMI 1649 + GlutaMAX (GIBCO Life Technologies, Carlsbad, California, USA), supplemented with $10 \% \mathrm{FCS}, 100 \mathrm{U} / \mathrm{mL}$ penicillin, $100 \mu \mathrm{g} / \mathrm{mL}$ streptomycin, and 50 $\mu \mathrm{M} \beta$-mercaptoethanol.

The purified naïve $\mathrm{T}$ cells were stimulated with $1 \mu \mathrm{g} / \mathrm{mL}$ plate-bound- $\alpha \mathrm{CD} 3$ (clone $145-2 \mathrm{C} 11$ ) and $1 \mu \mathrm{g} / \mathrm{mL}$ plate-bound- $\alpha \mathrm{CD} 28$ (clone 37.51) under Th2 cell polarized conditions $[10 \mathrm{ng} / \mathrm{mL}$ recombinant IL2, $30 \mathrm{ng} / \mathrm{mL}$ recombinant IL4, $10 \mu \mathrm{g} / \mathrm{mL} \alpha \mathrm{IL} 12$ (clone C17.8), and $10 \mu \mathrm{g} / \mathrm{mL} \alpha \mathrm{IFN} \gamma$ (clone AN18.17.24)] for 5, 12, 24, and $48 \mathrm{~h}$. The polarization of these Th2 cells was checked by intracellular staining of the master TF GATA3 for the 24-h (19\% GATA3 producers) and 48-h cultures (85\% GATA3 producers). After cultured cells were sorted for living cells using DAPI, mRNA was prepared using an RNeasy Plus Mini Kit (Qiagen, Hilden, Germany) with a gDNA degradation step. Finally, the RNA library was prepared from up to $3 \mu \mathrm{g}$ RNA using a TrueSeq Stranded mRNA Kit (Illumina, San Diego, California, USA) and sequenced in an Illumina HiSeq system.

The quality of the extracted sequence reads was checked, and sequences were trimmed using a Solexa trimming tool. The trimmed sequence reads where processed as described for the data from $\mathrm{Hu}$ et al. (2013) (vide supra). Expression and log2(fold change) values are listed in $\underline{\mathbf{S 2} \text { Table. }}$.

\section{Gene regulatory network assembly}

All preprocessed datasets were integrated in a large connectivity matrix describing the network. The initial network was established based on STAT6 and the 100 TFs, cytokines, and cytokine receptors differentially expressed in Stat6-KO RNA-seq experiments. Next, STAT6bound genes from the ChIP-seq dataset were added.

Subsequently, the genes were connected to STAT6 based on their expression in the Th2-RNA-seq experiments and annotated based on the expression information in other Th cell subtypes (RNA-seq, microarray experiments). Figure 2 illustrates the final gene regulatory network visualized with Cytoscape visualization and integration software (http://www. cytoscape.org/).

\section{Literature search of STAT6-regulated and STAT6-bound genes encoding TFs}

A literature search in (http://www.ncbi.nlm.nih.gov/pubmed) was carried out in February 2015 with the search phrase $<$ NAME_OF_TF $>$ AND ("t cell" OR "differentiation") for the 32 selected STAT6-regulated and STAT6-bound genes encoding TFs. The result lists were manually scanned to create Table 1, which describes the main function of each TF. Depending on this function, TFs were categorized in three groups: i) associated with differentiation of Th2 cells, ii) associated with differentiation of other Th cell subtypes, and iii) not associated with differentiation of Th cells. 


\section{Gene expression preferences of STAT6-regulated genes}

We characterized the STAT6-regulated genes using expression levels from the published microarray dataset (Wei et al., 2009) for the 10-day-old Th cell subtypes Th2, Th1, Th17, and iTreg. Those genes among the 100 STAT6-regulated genes with differential expression in only one cell subtype were detected by calculating the log2(fold change) between the different Th cell subtypes. The threshold for differentially expressed genes was set at 1.4fold $[\log 2$ (fold change) $=0.5]$. For example, a gene expressed at least 1.4 -fold higher in Th2 cells than in Th1, Th17, and iTreg cells was assigned to the "Th2 $>$ Th1 \& Th2 $>$ Th17 \& Th2 $>$ iTreg" category. Only 64 of the 100 STAT6-regulated genes were preferentially expressed in one cell subtype (Figure 3A). The log2(fold change) values and corresponding expression preferences are listed in $\underline{\mathbf{S 3} \text { Table. }}$.

\section{Asthma risk genes within the STAT6-regulated genes}

A list of 125 asthma risk genes was extracted by meta-analysis of SNPs from Renkonen et al. (2010) and filtered for TFs, cytokines, and cytokine receptors (Wei et

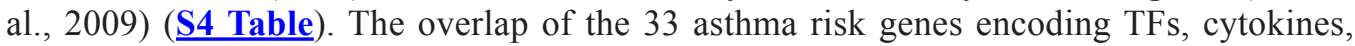
or cytokine receptors with the 100 STAT6-regulated genes in Th2 cells and with the 274 STAT4-regulated genes in Th1 cells (Vahedi et al., 2012) is shown in Figure 3B.

\section{Conflicts of interest}

The authors declare no conflict of interest.

\section{ACKNOWLEDGMENTS}

Research supported by the "e:Bio - Innovationswettbewerb Systembiologie" program from the German Federal Ministry of Education and Research (T-Sys to R. Baumgrass, U. Leser, and W. Chen).

\section{REFERENCES}

Ahlfors H, Limaye A, Elo LL, Tuomela S, et al. (2010). SATB1 dictates expression of multiple genes including IL-5 involved in human T helper cell differentiation. Blood 116: 1443-1453. http://dx.doi.org/10.1182/blood-2009-11-252205

Anders S and Huber W (2010). Differential expression analysis for sequence count data. Genome Biol. 11: R106. http:// dx.doi.org/10.1186/gb-2010-11-10-r106

Anders S, Pyl PT and Huber W (2015). HTSeq--a Python framework to work with high-throughput sequencing data. Bioinformatics 31: 166-169. http://dx.doi.org/10.1093/bioinformatics/btu638

Athey BD, Braxenthaler M, Haas M and Guo Y (2013). tranSMART: an open source and community-driven informatics and data sharing platform for clinical and translational research. AMIA Jt. Summits Transl. Sci. Proc. 2013: 6-8.

Bielekova B, Vodovotz Y, An G and Hallenbeck J (2014). How implementation of systems biology into clinical trials accelerates understanding of diseases. Front. Neurol. 5: 102. http://dx.doi.org/10.3389/fneur.2014.00102

Bonecchi R, Bianchi G, Bordignon PP, D’Ambrosio D, et al. (1998). Differential expression of chemokine receptors and chemotactic responsiveness of type 1 T helper cells (Th1s) and Th2s. J. Exp. Med. 187: 129-134. http://dx.doi. org/10.1084/jem.187.1.129

Brunner C, Sindrilaru A, Girkontaite I, Fischer KD, et al. (2007). BOB.1/OBF.1 controls the balance of TH1 and TH2 immune responses. EMBO J. 26: 3191-3202. http://dx.doi.org/10.1038/sj.emboj.7601742 
Chen J, Amasaki Y, Kamogawa Y, Nagoya M, et al. (2003a). Role of NFATx (NFAT4/NFATc3) in expression of immunoregulatory genes in murine peripheral CD4+ T cells. J. Immunol. 170: 3109-3117. http://dx.doi.org/10.4049/ ¡immunol.170.6.3109

Chen Y, Haviernik P, Bunting KD and Yang YC (2007). Cited2 is required for normal hematopoiesis in the murine fetal liver. Blood 110: 2889-2898. http://dx.doi.org/10.1182/blood-2007-01-066316

Chen Z, Lund R, Aittokallio T, Kosonen M, et al. (2003b). Identification of novel IL-4/Stat6-regulated genes in T lymphocytes. J. Immunol. 171: 3627-3635. http://dx.doi.org/10.4049/jimmunol.171.7.3627

Chen X, Rao SQ, Gao BH and Jiang ZQ (2015). Effect of early vitamin D supplementation on asthma and the possible mechanisms. Genet. Mol. Res. 14: 14136-14143. http://dx.doi.org/10.4238/2015.October.29.35

Chumakov AM, Silla A, Williamson EA and Koeffler HP (2007). Modulation of DNA binding properties of CCAAT/ enhancer binding protein epsilon by heterodimer formation and interactions with NFkappaB pathway. Blood 109: 4209-4219. http://dx.doi.org/10.1182/blood-2005-09-031963

Ciofani M, Madar A, Galan C, Sellars M, et al. (2012). A validated regulatory network for Th17 cell specification. Cell 151: 289-303. http://dx.doi.org/10.1016/j.cell.2012.09.016

Corcoran L, Emslie D, Kratina T, Shi W, et al. (2014). Oct2 and Obf1 as facilitators of B:T cell collaboration during a humoral immune response. Front. Immunol. 5: 108. http://dx.doi.org/10.3389/fimmu.2014.00108

Cron RQ, Zhou B, Brunvand MW and Lewis DB (2001). Octamer proteins inhibit IL-4 gene transcription in normal human CD4 T cells. Genes Immun. 2: 464-468. http://dx.doi.org/10.1038/sj.gene.6363811

D’Ambrosio D, Iellem A, Bonecchi R, Mazzeo D, et al. (1998). Selective up-regulation of chemokine receptors CCR4 and CCR8 upon activation of polarized human type 2 Th cells. J. Immunol. 161: 5111-5115.

da Rocha Junior LF, Rêgo MJ, Cavalcanti MB, Pereira MC, et al. (2013). Synthesis of a novel thiazolidinedione and evaluation of its modulatory effect on IFN- $\gamma$, IL-6, IL-17A, and IL-22 production in PBMCs from rheumatoid arthritis patients. BioMed Res. Int. 2013: 926060. http://dx.doi.org/10.1155/2013/926060

Del Prete G (1992). Human Th1 and Th2 lymphocytes: their role in the pathophysiology of atopy. Allergy 47: 450-455. http://dx.doi.org/10.1111/j.1398-9995.1992.tb00662.x

Dolhain RJ, van der Heiden AN, ter Haar NT, Breedveld FC, et al. (1996). Shift toward T lymphocytes with a T helper 1 cytokine-secretion profile in the joints of patients with rheumatoid arthritis. Arthritis Rheum. 39: 1961-1969. http:// dx.doi.org/10.1002/art.1780391204

Edgar R, Domrachev M and Lash AE (2002). Gene Expression Omnibus: NCBI gene expression and hybridization array data repository. Nucleic Acids Res. 30: 207-210. http://dx.doi.org/10.1093/nar/30.1.207

Elo LL, Järvenpää H, Tuomela S, Raghav S, et al. (2010). Genome-wide profiling of interleukin-4 and STAT6 transcription factor regulation of human Th2 cell programming. Immunity 32: 852-862. http://dx.doi.org/10.1016/j. immuni.2010.06.011

Elser B, Lohoff M, Kock S, Giaisi M, et al. (2002). IFN-gamma represses IL-4 expression via IRF-1 and IRF-2. Immunity 17: 703-712. http://dx.doi.org/10.1016/S1074-7613(02)00471-5

Farrar JD, Smith JD, Murphy TL, Leung S, et al. (2000). Selective loss of type I interferon-induced STAT4 activation caused by a minisatellite insertion in mouse Stat2. Nat. Immunol. 1: 65-69. http://dx.doi.org/10.1038/76932

Grégoire JM and Roméo PH (1999). T-cell expression of the human GATA-3 gene is regulated by a non-lineage-specific silencer. J. Biol. Chem. 274: 6567-6578. http://dx.doi.org/10.1074/jbc.274.10.6567

Guibal FC, Moog-Lutz C, Smolewski P, Di Gioia Y, et al. (2002). ASB-2 inhibits growth and promotes commitment in myeloid leukemia cells. J. Biol. Chem. 277: 218-224. http://dx.doi.org/10.1074/jbc.M108476200

Han S, Nam J, Li Y, Kim S, et al. (2010). Regulation of dendritic spines, spatial memory, and embryonic development by the TANC family of PSD-95-interacting proteins. J. Neurosci. 30: 15102-15112. http://dx.doi.org/10.1523/ JNEUROSCI.3128-10.2010

Hinks A, Eyre S, Ke X, Barton A, et al.; Childhood Arthritis Prospective Study; UKRAG Consortium; BSPAR Study Group (2010). Association of the AFF3 gene and IL2/IL21 gene region with juvenile idiopathic arthritis. Genes Immun. 11: 194-198. http://dx.doi.org/10.1038/gene.2009.105

Honda K, Yanai H, Negishi H, Asagiri M, et al. (2005). IRF-7 is the master regulator of type-I interferon-dependent immune responses. Nature 434: 772-777. http://dx.doi.org/10.1038/nature03464

Hu G, Tang Q, Sharma S, Yu F, et al. (2013). Expression and regulation of intergenic long noncoding RNAs during T cell development and differentiation. Nat. Immunol. 14: 1190-1198. http://dx.doi.org/10.1038/ni.2712

Hudson NJ, Reverter A, Greenwood PL, Guo B, et al. (2015). Longitudinal muscle gene expression patterns associated with differential intramuscular fat in cattle. Animal 9: 650-659. http://dx.doi.org/10.1017/S1751731114002754

Imamura K, Maeda S, Kawamura I, Matsuyama K, et al. (2014). Human immunodeficiency virus type 1 enhancer-binding protein 3 is essential for the expression of asparagine-linked glycosylation 2 in the regulation of osteoblast and

Genetics and Molecular Research 15 (2): gmr.15028493

CFUNPEC-RP www.funpecrp.com.br 
chondrocyte differentiation. J. Biol. Chem. 289: 9865-9879. http://dx.doi.org/10.1074/jbc.M113.520585

Jabeen R, Goswami R, Awe O, Kulkarni A, et al. (2013). Th9 cell development requires a BATF-regulated transcriptional network. J. Clin. Invest. 123: 4641-4653. http://dx.doi.org/10.1172/JCI69489

Ji H, Jiang H, Ma W, Johnson DS, et al. (2008). An integrated software system for analyzing ChIP-chip and ChIP-seq data. Nat. Biotechnol. 26: 1293-1300. http://dx.doi.org/10.1038/nbt.1505

Karnowski A, Chevrier S, Belz GT, Mount A, et al. (2012). B and T cells collaborate in antiviral responses via IL-6, IL-21, and transcriptional activator and coactivator, Oct2 and OBF-1. J. Exp. Med. 209: 2049-2064. http://dx.doi. org/10.1084/jem.20111504

Kashiwada M, Cassel SL, Colgan JD and Rothman PB (2011). NFIL3/E4BP4 controls type 2 T helper cell cytokine expression. EMBO J. 30: 2071-2082. http://dx.doi.org/10.1038/emboj.2011.111

Kay AB (2001). Allergy and allergic diseases. Second of two parts. N. Engl. J. Med. 344: 109-113. http://dx.doi. org/10.1056/NEJM200101113440206

Kim D, Pertea G, Trapnell C, Pimentel H, et al. (2013). TopHat2: accurate alignment of transcriptomes in the presence of insertions, deletions and gene fusions. Genome Biol. 14: R36. http://dx.doi.org/10.1186/gb-2013-14-4-r36

Kissick HT, Sanda MG, Dunn LK, Pellegrini KL, et al. (2014). Androgens alter T-cell immunity by inhibiting T-helper 1 differentiation. Proc. Natl. Acad. Sci. USA 111: 9887-9892. http://dx.doi.org/10.1073/pnas.1402468111

Koga T, Hedrich CM, Mizui M, Yoshida N, et al. (2014). CaMK4-dependent activation of AKT/mTOR and CREM- $\alpha$ underlies autoimmunity-associated Th17 imbalance. J. Clin. Invest. 124: 2234-2245. http://dx.doi.org/10.1172/ JCI73411

König R, Stertz S, Zhou Y, Inoue A, et al. (2010). Human host factors required for influenza virus replication. Nature 463: 813-817. http://dx.doi.org/10.1038/nature08699

Kwon SJ, Crespo-Barreto J, Zhang W, Wang T, et al. (2014). KLF13 cooperates with c-Maf to regulate IL-4 expression in CD4+ T cells. J. Immunol. 192: 5703-5709. http://dx.doi.org/10.4049/jimmunol.1302830

Lai CY, Lin SY, Wu CK, Yeh LT, et al. (2012). Tyrosine phosphorylation of c-Maf enhances the expression of IL-4 gene. J. Immunol. 189: 1545-1550. http://dx.doi.org/10.4049/jimmunol.1200405

Laurence A, Amarnath S, Mariotti J, Kim YC, et al. (2012). STAT3 transcription factor promotes instability of nTreg cells and limits generation of iTreg cells during acute murine graft-versus-host disease. Immunity 37: 209-222. http:// dx.doi.org/10.1016/j.immuni.2012.05.027

Li H and Durbin R (2009). Fast and accurate short read alignment with Burrows-Wheeler transform. Bioinformatics 25: 1754-1760. http://dx.doi.org/10.1093/bioinformatics/btp324

Li H, Handsaker B, Wysoker A, Fennell T, et al.; 1000 Genome Project Data Processing Subgroup (2009). The Sequence Alignment/Map format and SAMtools. Bioinformatics 25: 2078-2079. http://dx.doi.org/10.1093/bioinformatics/ $\underline{\operatorname{btp} 352}$

Litvak V, Ratushny AV, Lampano AE, Schmitz F, et al. (2012). A FOXO3-IRF7 gene regulatory circuit limits inflammatory sequelae of antiviral responses. Nature 490: 421-425. http://dx.doi.org/10.1038/nature11428

Liu Q, Boudot A, Ni J, Hennessey T, et al. (2014). Cyclin D1 and C/EBP $\beta$ LAP1 operate in a common pathway to promote mammary epithelial cell differentiation. Mol. Cell. Biol. 34: 3168-3179. http://dx.doi.org/10.1128/MCB.00039-14

Liu X, Nurieva RI and Dong C (2013). Transcriptional regulation of follicular T-helper (Tfh) cells. Immunol. Rev. 252: 139-145. http://dx.doi.org/10.1111/imr.12040

Lohoff M and Mak TW (2005). Roles of interferon-regulatory factors in T-helper-cell differentiation. Nat. Rev. Immunol. 5: 125-135. http://dx.doi.org/10.1038/nri1552

Lund R, Ahlfors H, Kainonen E, Lahesmaa AM, et al. (2005). Identification of genes involved in the initiation of human Th1 or Th2 cell commitment. Eur. J. Immunol. 35: 3307-3319. http://dx.doi.org/10.1002/eji.200526079

Ma J, Wang R, Fang X, Ding Y, et al. (2011). Critical role of TCF-1 in repression of the IL-17 gene. PLoS One 6: e24768. http://dx.doi.org/10.1371/journal.pone.0024768

Maruyama S, Sumita K, Shen H, Kanoh M, et al. (2003). Identification of IFN regulatory factor-1 binding site in IL-12 p40 gene promoter. J. Immunol. 170: 997-1001. http://dx.doi.org/10.4049/jimmunol.170.2.997

Mason JL, Angelastro JM, Ignatova TN, Kukekov VG, et al. (2005). ATF5 regulates the proliferation and differentiation of oligodendrocytes. Mol. Cell. Neurosci. 29: 372-380. http://dx.doi.org/10.1016/j.mcn.2005.03.004

Nie L, Zhao Y, Wu W, Yang YZ, et al. (2011). Notch-induced Asb2 expression promotes protein ubiquitination by forming non-canonical E3 ligase complexes. Cell Res. 21: 754-769. http://dx.doi.org/10.1038/cr.2010.165

Notani D, Gottimukkala KP, Jayani RS, Limaye AS, et al. (2010). Global regulator SATB1 recruits beta-catenin and regulates $\mathrm{T}(\mathrm{H}) 2$ differentiation in Wnt-dependent manner. PLoS Biol. 8: e1000296. http://dx.doi.org/10.1371/ journal.pbio.1000296

Novershtern N, Subramanian A, Lawton LN, Mak RH, et al. (2011). Densely interconnected transcriptional circuits 
control cell states in human hematopoiesis. Cell 144: 296-309. http://dx.doi.org/10.1016/j.cell.2011.01.004

Ouyang X, Zhang R, Yang J, Li Q, et al. (2011). Transcription factor IRF8 directs a silencing programme for TH17 cell differentiation. Nat. Commun. 2: 314. http://dx.doi.org/10.1038/ncomms1311

Pabbisetty SK, Rabacal W, Maseda D, Cendron D, et al. (2014). KLF2 is a rate-limiting transcription factor that can be targeted to enhance regulatory T-cell production. Proc. Natl. Acad. Sci. USA 111: 9579-9584. http://dx.doi. org/10.1073/pnas.1323493111

Palazon A, Goldrath AW, Nizet V and Johnson RS (2014). HIF transcription factors, inflammation, and immunity. Immunity 41: 518-528. http://dx.doi.org/10.1016/j.immuni.2014.09.008

Ramsey SA, Klemm SL, Zak DE, Kennedy KA, et al. (2008). Uncovering a macrophage transcriptional program by integrating evidence from motif scanning and expression dynamics. PLOS Comput. Biol. 4: e1000021. http://dx.doi. org/10.1371/journal.pcbi.1000021

Rengarajan J, Tang B and Glimcher LH (2002). NFATc2 and NFATc3 regulate T(H)2 differentiation and modulate TCRresponsiveness of naïve T(H)cells. Nat. Immunol. 3: 48-54. http://dx.doi.org/10.1038/ni744

Renkonen J, Joenväärä S, Parviainen V, Mattila P, et al. (2010). Network analysis of single nucleotide polymorphisms in asthma. J. Asthma Allergy 3: 177-186. http://dx.doi.org/10.2147/JAA.S14459

Ribeiro de Almeida C, Heath H, Krpic S, Dingjan GM, et al. (2009). Critical role for the transcription regulator CCCTCbinding factor in the control of Th2 cytokine expression. J. Immunol. 182: 999-1010. http://dx.doi.org/10.4049/ ¡immunol.182.2.999

Richardson MW, Jadlowsky J, Didigu CA, Doms RW, et al. (2012). Kruppel-like factor 2 modulates CCR5 expression and susceptibility to HIV-1 infection. J. Immunol. 189: 3815-3821. http://dx.doi.org/10.4049/jimmunol.1201431

Rozenberg JM, Bhattacharya P, Chatterjee R, Glass K, et al. (2013). Combinatorial recruitment of CREB, C/EBP $\beta$ and c-Jun determines activation of promoters upon keratinocyte differentiation. PLoS One 8: e78179. http://dx.doi. org/10.1371/journal.pone.0078179

Saito A, Kanemoto S, Zhang Y, Asada R, et al. (2014). Chondrocyte proliferation regulated by secreted luminal domain of ER stress transducer BBF2H7/CREB3L2. Mol. Cell 53: 127-139. http://dx.doi.org/10.1016/j.molcel.2013.11.008

Salkowski CA, Kopydlowski K, Blanco J, Cody MJ, et al. (1999). IL-12 is dysregulated in macrophages from IRF-1 and IRF-2 knockout mice. J. Immunol. 163: 1529-1536.

Schraml BU, Hildner K, Ise W, Lee WL, et al. (2009). The AP-1 transcription factor Batf controls T(H)17 differentiation. Nature 460: 405-409.

Schuster SC (2008). Next-generation sequencing transforms today's biology. Nat. Methods 5: 16-18. http://dx.doi. org/10.1038/nmeth1156

Searls DB (2005). Data integration: challenges for drug discovery. Nat. Rev. Drug Discov. 4: 45-58. http://dx.doi. org $/ 10.1038 / \mathrm{nrd} 1608$

Slater T, Bouton C and Huang ES (2008). Beyond data integration. Drug Discov. Today 13: 584-589. http://dx.doi. org/10.1016/j.drudis.2008.01.008

Stritesky GL, Muthukrishnan R, Sehra S, Goswami R, et al. (2011). The transcription factor STAT3 is required for T helper 2 cell development. Immunity 34: 39-49. http://dx.doi.org/10.1016/j.immuni.2010.12.013

Subramanian N, Torabi-Parizi P, Gottschalk RA, Germain RN, et al. (2015). Network representations of immune system complexity. Wiley Interdiscip. Rev. Syst. Biol. Med. 7: 13-38. http://dx.doi.org/10.1002/wsbm.1288

Taki S, Sato T, Ogasawara K, Fukuda T, et al. (1997). Multistage regulation of Th1-type immune responses by the transcription factor IRF-1. Immunity 6: 673-679. http://dx.doi.org/10.1016/S1074-7613(00)80443-4

Tan RJ, Gibbons LJ, Potter C, Hyrich KL, et al.; BRAGGSS (2010). Investigation of rheumatoid arthritis susceptibility genes identifies association of AFF3 and CD226 variants with response to anti-tumour necrosis factor treatment. Ann. Rheum. Dis. 69: 1029-1035. http://dx.doi.org/10.1136/ard.2009.118406

Tanaka S, Suto A, Iwamoto T, Kashiwakuma D, et al. (2014). Sox5 and c-Maf cooperatively induce Th17 cell differentiation via RORgt induction as downstream targets of Stat3. J. Exp. Med. 211: 1857-1874. http://dx.doi. org/10.1084/jem.20130791

Umemura M, Tsunematsu K, Shimizu YI, Nakano H, et al. (2015). Activating transcription factor 5 is required for mouse olfactory bulb development via interneuron. Biosci. Biotechnol. Biochem. 79: 1082-1089. http://dx.doi.org/10.1080 /09168451.2015.1012042

Ushijima T, Okazaki K, Tsushima H and Iwamoto Y (2014). CCAAT/enhancer-binding protein $\beta$ regulates the repression of type II collagen expression during the differentiation from proliferative to hypertrophic chondrocytes. J. Biol. Chem. 289: 2852-2863. http://dx.doi.org/10.1074/jbc.M113.492843

Vahedi G, Takahashi H, Nakayamada S, Sun HW, et al. (2012). STATs shape the active enhancer landscape of T cell populations. Cell 151: 981-993. http://dx.doi.org/10.1016/j.cell.2012.09.044 
Wei G, Wei L, Zhu J, Zang C, et al. (2009). Global mapping of H3K4me3 and H3K27me3 reveals specificity and plasticity in lineage fate determination of differentiating CD4+ T cells. Immunity 30: 155-167. http://dx.doi.org/10.1016/j. immuni.2008.12.009

Wei L, Vahedi G, Sun HW, Watford WT, et al. (2010). Discrete roles of STAT4 and STAT6 transcription factors in tuning epigenetic modifications and transcription during T helper cell differentiation. Immunity 32: 840-851. http://dx.doi. org/10.1016/j.immuni.2010.06.003

Wingender E, Dietze P, Karas H and Knüppel R (1996). TRANSFAC: a database on transcription factors and their DNA binding sites. Nucleic Acids Res. 24: 238-241. http://dx.doi.org/10.1093/nar/24.1.238

Wu J and Lingrel JB (2005). Krüppel-like factor 2, a novel immediate-early transcriptional factor, regulates IL-2 expression in T lymphocyte activation. J. Immunol. 175: 3060-3066. http://dx.doi.org/10.4049/jimmunol.175.5.3060

Wuelling M, Kaiser FJ, Buelens LA, Braunholz D, et al. (2009). Trps1, a regulator of chondrocyte proliferation and differentiation, interacts with the activator form of Gli3. Dev. Biol. 328: 40-53. http://dx.doi.org/10.1016/j. ydbio.2009.01.012

Yamane $\mathrm{H}$ and Paul WE (2013). Early signaling events that underlie fate decisions of naive CD4(+) T cells toward distinct T-helper cell subsets. Immunol. Rev. 252: 12-23. http://dx.doi.org/10.1111/imr.12032

Yi H, Guo C, Yu X, Zuo D, et al. (2012). Mouse CD11b+Gr-1+ myeloid cells can promote Th17 cell differentiation and experimental autoimmune encephalomyelitis. J. Immunol. 189: 4295-4304. http://dx.doi.org/10.4049/ jimmunol.1200086

Yosef N, Shalek AK, Gaublomme JT, Jin H, et al. (2013). Dynamic regulatory network controlling TH17 cell differentiation. Nature 496: 461-468. http://dx.doi.org/10.1038/nature11981

Yu Q, Sharma A, Oh SY, Moon HG, et al. (2009). T cell factor 1 initiates the Thelper type 2 fate by inducing the transcription factor GATA-3 and repressing interferon-gamma. Nat. Immunol. 10: 992-999. http://dx.doi.org/10.1038/ni.1762

Yu X, Rollins D, Ruhn KA, Stubblefield JJ, et al. (2013). TH17 cell differentiation is regulated by the circadian clock. Science 342: 727-730. http://dx.doi.org/10.1126/science.1243884

Zhang R, Chen K, Peng L and Xiong H (2012). Regulation of T helper cell differentiation by interferon regulatory factor family members. Immunol. Res. 54: 169-176. http://dx.doi.org/10.1007/s12026-012-8328-0

Zhao Y, Zhang YD, Zhang YY, Qian SW, et al. (2014). p300-dependent acetylation of activating transcription factor 5 enhances C/EBP $\beta$ transactivation of C/EBP $\alpha$ during 3T3-L1 differentiation. Mol. Cell. Biol. 34: 315-324. http:// dx.doi.org/10.1128/MCB.00956-13

Zhu J and Paul WE (2010). Peripheral CD4+ T-cell differentiation regulated by networks of cytokines and transcription factors. Immunol. Rev. 238: 247-262. http://dx.doi.org/10.1111/j.1600-065X.2010.00951.x

\section{Supplementary material}

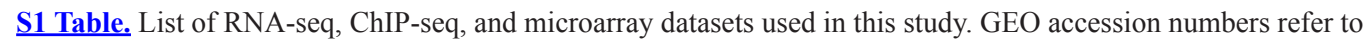
the original publication.

S2 Table. List of raw data (expression and $\log 2$ (fold change) values) used for construction of the global gene regulatory STAT6 network.

S3 Table. Log2(fold change) values and corresponding expression preferences for the 100 STAT6-regulated genes.

S4 Table. List of the 125 SNP-associated asthma risk genes. Additionally, asthma risk genes within the STAT6regulated genes in Th2 cells and STAT4-regulated genes in Th1 cells are shown. 